\title{
Commentary on "Effect of an exercise programme for reducing abdominal fat on overactive bladder symptoms in young overweight women"
}

\author{
Abdelmageed Abdelrahman ${ }^{1}$ \\ Received: 11 November 2019 / Accepted: 14 November 2019 / Published online: 17 December 2019 \\ (C) The International Urogynecological Association 2019
}

This study [1] was aimed at assessing the effect of exercise on overactive bladder symptoms in young women. The authors had a sample of 70 women in their study; 36 women receiving treatment and 34 women in the control group. The training programme had elements of aerobic training (stationary bicycle for $20 \mathrm{~min}$ ), dynamic warm-up (stretching muscles by slow and controlled movements for $10 \mathrm{~min}$ ), strength training for a reduction of abdominal fat (deep abdominal muscle activation for $20 \mathrm{~min}$ and strengthening of superficial abdominal muscles), and static stretching (passive stretching of lower limbs and abdominal muscles for $10 \mathrm{~min}$ ).

Overactive bladder symptoms were evaluated using a voiding diary, the Overactive Bladder Questionnaire (OABQ) and the Patient Perception of Intensity of Urgency Scale (PPIUS). The intervention used was a programme for the reduction of abdominal fat, with elements of aerobic training, strengthening of the abdominal muscles, and stretching. Inclusion criteria included women aged 18-35, BMI 2529.9 , waist circumference above $88 \mathrm{~cm}$, urgency (usually accompanied by frequency and nocturia), with or without urge urinary incontinence. Exclusion criteria included the presence of stress urinary incontinence symptoms, surgical treatment of gynaecological, urological, oncological and neurological diseases, urinary tract infection, and incomplete questionnaires.
The authors concluded that there was a reduction in overactive bladder symptoms objectively confirmed following the 12-week programme for the reduction of abdominal fat.

Strengths of this study include the high control of the exercise interventions and the short duration of the training programme. This has the added advantage of ensuring compliance with the programme. Limitations of the study include the lack of strict control of calories in both groups; furthermore, no specific diet was recommended to the study participants.

\section{Compliance with ethical standards}

Conflicts of interest None.

\section{Reference}

1. Hagovska M, Švihra J, Buková A, Dračková D, Horbacz A, Nagyová I. Effect of an exercise programme for reducing abdominal fat on overactive bladder symptoms in young overweight women.Int Urogynecol J. 2019. https://doi.org/10.1007/s00192-019-04157-8.

Publisher's note Springer Nature remains neutral with regard to jurisdictional claims in published maps and institutional affiliations.

Abdelmageed Abdelrahman

abdelmageed@hotmail.co.uk

1 Department of Urogynaecology, Liverpool Women's Hospital NHS Foundation Trust, Liverpool, UK 